\title{
Phytodiversity of the remnants of Canarian endemic juniper woodlands on El Hierro, Canary Islands
}

\author{
La phytodiversité dans les rémanents \\ de la forêt endémique de genévrier \\ d'El Hierro, îles Canaries
}

\author{
Angel ROMO ${ }^{1,3}$, Montserrat SALVÀ-CATARINEU ${ }^{2,3}$ \\ 1. Botanical Institute of Barcelona, Consejo Superior de Investigaciones Científicas, \\ IBB-CSIC-ICUB, Passeig del Migdia s/n., 08038 Barcelona, Spain \\ E-mail : a.romo@ibb.csic.es \\ 2. Departament de Geografia Física i Anàlisi Geogràfica Regional, \\ Universitat de Barcelona, Montalegre 6, 08001 Barcelona, Spain \\ E-mail : salva@ub.edu \\ 3. Grup de Recerca Paisatge i Paleoambients a la muntanya mediterrània
}

\begin{abstract}
Hot spots of endemicity such as the Canary Isles are of particular interest as far as conserving biodiversity is concerned. The most westerly of these oceanic islands preserve dry woodlands dominated by the Canarian juniper (Juniperus turbinata subsp. canariensis). The island of EI Hierro has been studied because it conserves the biggest extension of this kind of dry forest in the Canary Islands. In this context, the arid zones are reputed as being zones that present a low biodiversity.

A study of 24 plots of $200 \mathrm{~m}^{2}$ has been carried out. All the vascular taxa present have been identified, and the degree of cover and gregarianism has been calculated using the BraunBlanquet methodology. For each one of the taxa the biological type and the chorology have been assigned. Tables with relevés have been elaborated and an analysis of clusters and dendrograms to see the affinities between the studied plots has been drawn up.

Although less than a tenth part of the natural vegetation is composed of juniper woodlands dominated by Juniperus turbinata subsp. canariensis, it contains more than a fifth of the total of wild vascular plants from the island. This flora associated with junipers contains $30,6 \%$ of the endemic taxa from the Macaronesian region. Between them it represents $22,1 \%$ of
\end{abstract}

Keywords: dendrogram, dry forest, endemicity, hot-spot, Macaronesian. endemics from the Canary Islands, 4,6\% of endemics from Canary islands and Madeira, $3,1 \%$ of endemics for El Hierro and $0,8 \%$ of endemics from the Canary, Madeira and Cape Verde islands.

The analysis of phytodiversity of the different plots studied reflects a high affinity between the dry juniper woodlands from the lowlands and the others situated in more elevated places. From these we can differentiate those situated in moister conditions, which enter in contact with laurel forests on one hand, and those in close proximity with the Pinus canariensis forest, on the other.

\section{Resumé}

Les points chauds d'endémicité, tels que le sont les îles Canaries, sont particulièrement intéressants pour la conservation de la biodiversité. Les îles les plus occidentales des Canaries préservent des forêts sèches dominées par le genévrier Juniperus turbinata subsp. canariensis. Notre étude se concentre sur l'île d'El Hierro, parce qu'elle présente la plus grande extension de ce type de forêt sèche dans l'ensemble des îles Canaries. Dans ce contexte, les zones arides sont réputées pour avoir une faible biodiversité.

Nous avons étudié 24 parcelles de $200 \mathrm{~m}^{2}$, pour lesquelles toutes les plantes vasculaires ont été

Mots clés : dendrogramme, endémicité, forêt sèche, hot-spot, Macaronésie. 
identifiées, leur couverture et leur sociabilité ont été calculées en employant la méthodologie établie par Braun-Blanquet. Pour chaque taxon, le type biologique et la chorologie ont été précisés. Nous avons élaboré des tableaux avec l'ensemble des relevés et, afin de connaître les affinités des parcelles étudiées, nous présentons également les résultats sous forme de clusters et de dendrogrammes.

Bien que la forêt de genévriers (dominée par Juniperus turbinata subsp. canariensis) représente moins d'un dixième de la végétation naturelle, on y trouve un cinquième du total de la flore vasculaire de l'île d'El Hierro. La flore associée avec le genévrier comprend 30,6\% des taxons endémiques de la région macaronésique, parmi lesquels 22,1\% d'endémiques des îles Canaries, 4,6\% d'endémiques des îles Canaries et Madeira, 3,1\% d'endémiques d'El Hierro, et 0,8\% d'endémiques des îles Canaries, Madeira et Cap Vert.

L'analyse de la phytodiversité des différentes parcelles étudiées reflète une très forte affinité entre les forêts sèches de genévriers des parcelles les plus basses et les forêts de genévriers des parcelles de plus haute altitude. Parmi les parcelles plus élevées, on peut différencier celles situées dans des conditions plus humides près de la forêt laurifoliée, de celles situées à proximité des forêts de Pinus canariensis en ambiances plus sèches.

\section{Introduction}

The Canary Islands are a centre for plant biodiversity (Médail \& Quézel 1999). They are usually included in the Mediterranean hotspot, a biodiversity hotspot recognized at a world level (Blondel \& Aronson 1999; Myers et al. 2000). Within this archipelago our study is focused on the juniper woodlands from the island of El Hierro. It is on this island and Gomera island that the greater surfaces of juniper woodlands in the Canary archipelago are to be found (Fernández Palacios et al. 2008). These Canarian juniper woodlands, dominated by Juniperus turbinata Guss. subsp. canariensis (Guyot) RivasMart., Wildpret \& P. Pérez, have been defined as thermophilous woodlands (Santos 1980). They are located in low and middle areas "medianías" of the most westerly Canary islands (Otto et al. 2006). They are dry forests with a low tree canopy and, in fact, they are formed by canopy and intercanopy patches where nanophanerophytes, chamaephytes and therophytes grow. These woodlands have suffered considerable regression due to anthropic activity, and now only small fragments of them remain (Otto et al. 2006). Only a few phytosociological studies have been made on El Hierro's juniper woodlands, and these rather general and included within standard vegetation works (del Arco et al. 1996; von Gaisberg 2005). In this scenario we would like to answer the following questions: what level of phytodiversity do these woodland environments at present manifest? and which environmental factors are conditioning this biodiversity?

\section{Material and methods}

El Hierro island is located in the southwestern extreme of the Canary Isles, lying between $17^{\circ} 52^{\prime}-18^{\circ} 09^{\prime} \mathrm{W}$ and $27^{\circ} 38^{\prime}-$ $27^{\circ} 51^{\prime} \mathrm{N}$. It is a small island $\left(278.5 \mathrm{~km}^{2}\right)$, the most oceanic island of the Archipelago (Nogales et al. 2009), and geologically the youngest, being $1 \mathrm{M}$ yr of age (Fuster et al. 1993). In order to know the phytodiversity of these juniper woodlands 24 plots of $200 \mathrm{~m}^{2}$ have been studied, each plot being situated at different levels, aspects and slopes (Figure 1). The plots are found between $206 \mathrm{~m}$ and $1.012 \mathrm{~m}$ above see level. As well as the habitat conditions, all the vascular plants were identified in each plot, and their cover and degree of gregariousness were calculated; for this purpose the Braun-Blanquet (1979) method was employed. The life form and chorology of each taxon were then assigned (Stierstorfer \& von Gaisberg 2006). With this combined data two tables have been elaborated (Tables 1 and 2). One with the physical characteristics of the plots, and another with their phytodiversity. In order to establish the affinities between the studied plots, the results are presented in cluster and dendrogram form (Figure 5). Dendrograms were produced according to the unweighted pair-group mean arithmetic method (UPGMA), using B-Vegana software (De Cáceres et al. 2003).

\section{Results}

Although the total area of Juniperus turbinata subsp. canariensis woodland covers less than a tenth part of the natural vegetation of the island, within it is found more than a fifth of the total amount of the vascular flora of El Hierro. More than 131 (Table 2) out of the 


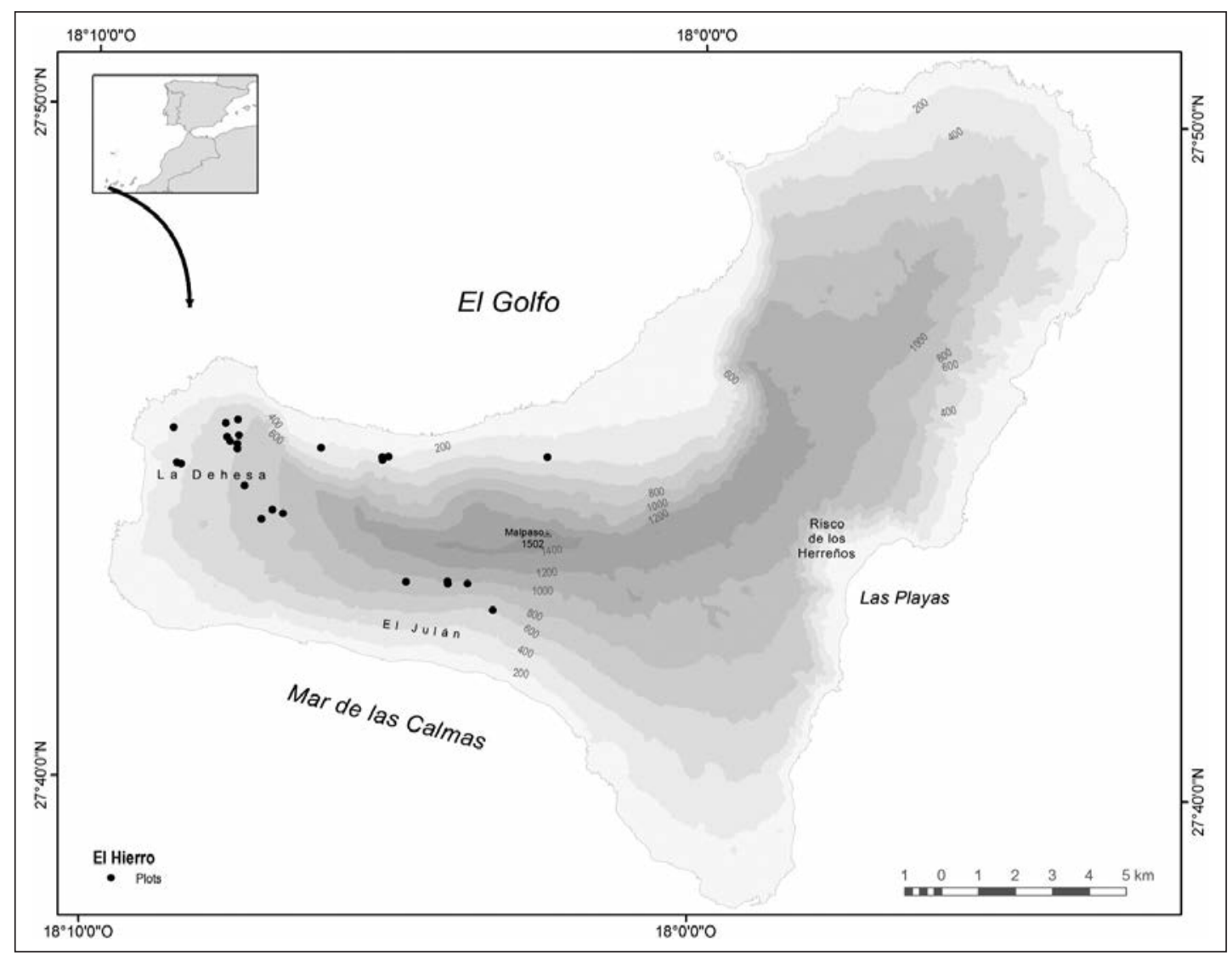

Figure 1 - Location of the studied plots on El Hierro island.

Table 1 - Characteristics of the plots studied.

\begin{tabular}{|c|c|c|c|c|c|c|c|}
\hline Plots & Code & Latitude & Longitude & Altitude (m) & Aspect & Slope $\left(^{\circ}\right)$ & Richness \\
\hline 1 [P_05]: El Cres & CR1 & $27^{\circ} 44^{\prime} 22^{\prime \prime} \mathrm{N}$ & $18^{\circ} 07^{\prime} 26^{\prime \prime} \mathrm{W}$ & 740 & WNW & 17 & 29 \\
\hline 2 [P_06]: El Cres & CR2 & $27^{\circ} 43^{\prime} 53^{\prime \prime} \mathrm{N}$ & $18^{\circ} 07^{\prime} 09^{\prime \prime} \mathrm{W}$ & 750 & $\mathrm{~N}$ & 31 & 26 \\
\hline 3 [P_07]: Barranco & & & & & & & \\
\hline de la Charca & $\mathrm{BC} 1$ & $27^{\circ} 44^{\prime} 41 " \mathrm{~N}$ & $18^{\circ} 08^{\prime} 34^{\prime \prime} \mathrm{W}$ & 342 & W & 14 & 15 \\
\hline 4 [P_08]: El Julán & JU2 & $27^{\circ} 42^{\prime} 59^{\prime \prime} \mathrm{N}$ & $18^{\circ} 03^{\prime} 43^{\prime \prime W}$ & 990 & SSW & 32 & 16 \\
\hline 5 [P_09]: Sabinosa & SA2 & $27^{\circ} 44^{\prime} 50^{\prime \prime} \mathrm{N}$ & $18^{\circ} 05^{\prime} 11^{\prime \prime W}$ & 254 & NNW & 35 & 24 \\
\hline 6 [P_10]: Sabinosa & SA1 & $27^{\circ} 44^{\prime} 51$ "N & $18^{\circ} 05^{\prime} 04^{\prime \prime} \mathrm{W}$ & 265 & NNE & 42 & 31 \\
\hline 7 [P_11]: Sabinosa & SA3 & $27^{\circ} 44^{\prime} 48^{\prime \prime} \mathrm{N}$ & $18^{\circ} 05^{\prime} 10^{\prime \prime} \mathrm{W}$ & 292 & NE & 40 & 16 \\
\hline 8 [P_12]: El Sabinar & SR6 & $27^{\circ} 44^{\prime} 55^{\prime \prime} \mathrm{N}$ & $18^{\circ} 07^{\prime} 34^{\prime \prime} \mathrm{W}$ & 608 & NW & 18 & 24 \\
\hline 9 [P_13]: El Sabinar & SR2 & $27^{\circ} 45^{\prime} 07^{\prime \prime} \mathrm{N}$ & $18^{\circ} 07^{\prime} 33^{\prime \prime W}$ & 589 & WNW & 20 & 28 \\
\hline 10 [P_14]: El Sabinar & SR5 & $27^{\circ} 44^{\prime} 59^{\prime \prime} \mathrm{N}$ & $18^{\circ} 07^{\prime} 35^{\prime \prime W}$ & 595 & W & 10 & 21 \\
\hline 11 [P_15]: El Julán & JU3 & $27^{\circ} 42^{\prime} 59^{\prime \prime} \mathrm{N}$ & $18^{\circ} 04^{\prime} 03^{\prime \prime} \mathrm{W}$ & 982 & S & 38 & 15 \\
\hline 12 [P_16]: El Julán & JU1 & $27^{\circ} 43^{\prime} 011^{\prime N} \mathrm{~N}$ & $18^{\circ} 04^{\prime} 04^{\prime \prime} \mathrm{W}$ & 1012 & S & 40 & 22 \\
\hline 13 [P_17]: El Sabinar & SR1 & $27^{\circ} 45^{\prime} 13^{\prime \prime} \mathrm{N}$ & $18^{\circ} 08^{\prime} 38^{\prime \prime W}$ & 304 & SE & 12 & 18 \\
\hline 14 [P_18]: Barranco & & & & & & & \\
\hline de la Charca & BC2 & $27^{\circ} 44^{\prime} 41^{\prime N} \mathrm{~N}$ & $18^{\circ} 08^{\prime} 30^{\prime \prime W}$ & 363 & NNW & 21 & 26 \\
\hline 15 [P_19]: El Sabinar & SR3 & $27^{\circ} 45^{\prime} 05^{\prime \prime N}$ & $18^{\circ} 07^{\prime} 45^{\prime \prime} \mathrm{W}$ & 536 & NW & 17 & 16 \\
\hline 16 [P_20]: El Sabinar & SR4 & $27^{\circ} 45^{\prime} 01 " \mathrm{~N}$ & $18^{\circ} 07^{\prime} 41^{\prime \prime W}$ & 505 & NNW & 19 & 14 \\
\hline 17 [P_21]: El Sabinar & SR7 & $27^{\circ} 45^{\prime} 17.6^{\prime \prime} \mathrm{N}$ & $18^{\circ} 07^{\prime} 46.4^{\prime \prime} W$ & 515 & NNE & 19 & 17 \\
\hline 18 [P_22]: El Sabinar & SR8 & $27^{\circ} 45^{\prime} 21.0^{\prime \prime} \mathrm{N}$ & $18^{\circ} 07^{\prime} 34.5^{\prime \prime} \mathrm{W}$ & 530 & NW & 10 & 21 \\
\hline 19 [P_23]: El Julán & $\mathrm{JU} 4$ & $27^{\circ} 43^{\prime} 00.2^{\prime \prime} \mathrm{N}$ & $18^{\circ} 04^{\prime} 44.3^{\prime \prime} \mathrm{W}$ & 947 & SSE & 20 & 16 \\
\hline 20 [P_24]: EL Julán & JU5 & $27^{\circ} 42^{\prime} 36.8^{\prime \prime} \mathrm{N}$ & $18^{\circ} 03^{\prime} 17.9^{\prime \prime} \mathrm{W}$ & 649 & W & 29 & 20 \\
\hline 21 [P_25]: El Cres & CR3 & $27^{\circ} 44^{\prime} 01.5^{\prime \prime} \mathrm{N}$ & $18^{\circ} 06^{\prime} 58.1$ "W & 776 & WSW & 12 & 31 \\
\hline 22 [P_26]: El Cres & CR4 & $27^{\circ} 43^{\prime} 58.4^{\prime \prime} \mathrm{N}$ & $18^{\circ} 06^{\prime} 47.6^{\prime \prime} \mathrm{W}$ & 817 & W & 14 & 31 \\
\hline 23 [P_27]: Sabinosa & SA4 & $27^{\circ} 44^{\prime} 57.6^{\prime \prime} \mathrm{N}$ & $18^{\circ} 06^{\prime} 11.5^{\prime \prime} \mathrm{W}$ & 206 & $\mathrm{~N}$ & 37 & 31 \\
\hline 24 [P_28]: Sabinosa & SA5 & $27^{\circ} 44^{\prime} 54.1^{\prime \prime} \mathrm{N}$ & $18^{\circ} 02^{\prime} 27.6^{\prime \prime} \mathrm{W}$ & 298 & $\mathrm{~N}$ & 42 & 33 \\
\hline
\end{tabular}


Table 2 - Vascular plants of the plots studied.

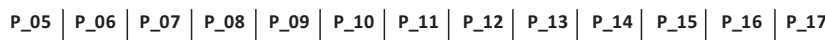
$\left[\begin{array}{lll|l|l|l|l|l|l|l|l|l|l}{[1]} & {[2]} & {[3]} & {[4]} & {[5]} & {[6]} & {[7]} & {[8]} & {[9]} & {[10]} & {[11]} & {[12]} & {[13]}\end{array}\right.$

Aeonium canariense Webb ex Bertel var. palmense (Webb ex Christ) H.Y. Liu Aira caryophyllea L. subsp. caryophyllea

Anagallis arvensis $\mathrm{L}$.

Arabidopsis thaliana (L.) Heynh.

Arenaria leptoclados (Reichenb.) Guss.

Argyranthemum hierrense

Artemisia thuscula Cav.

Asphodelus ramosus L. subsp. distalis Z. Diaz \& Valdes

Biserrula pelecinus $\mathrm{L}$.

Brachypodium distachyon (L.) Beauv.

Bromus diandrus Roth

Carlina salicifolia Cav.

Cheilanthes catanensis (Cosent.) H.P. Fuchs

Chenopodium murale $\mathrm{L}$.

Cistus monspeliensis L.

Cuscuta planiflora Ten.

Davallia canariensis (L.) Sm.

\begin{tabular}{l} 
Drusa glandulosa (Poir.) H. Wolf ex Engl. \\
\hline Echium hierense Webb ex Bolle
\end{tabular}

Echium hierrense Webb ex Bolle

Echium plantagineum $\mathrm{L}$.

Erodium cicutarium (L.) L'her. subsp. cicutarium

Eruca vesicaria (L.) Cav. subsp. vesicaria

Euphorbia balsamifera Ait.

Euphorbia lamarckii Sweet subsp. wildpretii (Molero \& Rovira) Rivas-Mart. \& Gaisberg

Galium parisiense L.

Geranium rotundifolium L.

Hyparrhenia sinaica (Delile) Llaurado ex G. Lopez

Ifloga spicata (Forskal) Schultz Bip. subsp. spicata

Juniperus turbinata Guss.

Kleinia neriifolia Haw.

Lagurus ovatus L.

Lathyrus articulatus $\mathrm{L}$.

Leontodon taraxacoides (Vill.) Merat subsp. taraxacoides

Lobularia canariensis (DC.) L. Borgen subsp. intermedia (Webb) L. Borgen

Matthiola incana (L.) R. Br. subsp. incana

Micromeria hyssopifolia Webb \& Berthel.

Misopates orontium (L.) Rafin.

Ononis dentata Solander ex Lowe

Paronychia canariensis (L.f.) Juss

Pericallis murrayi (Bornm.) B. Nord.

Periploca laevigata Aiton subsp. laevigata

Petrorhagia nanteuilii (Burnat) P.W. Ball \& Heywood

Phagnalon umbelliforme DC.

Plantago lagopus L.

Polycarpaea divaricata (Aiton) Poir.

Polycarpon tetraphyllum (L.) L.

Psoralea bituminosa $\mathrm{L}$.

Rubia fruticosa Ait.

Rumex bucephalophorus L. subsp. canariensis (Steinh.) Rech. f.

Rumex lunaria $\mathrm{L}$.

Sagina apetala Ard. subsp. apetala

Schizogyne sericea DC.

Senecio incrassatus Lowe

Silene gallica $\mathrm{L}$.

Sisymbrium erysimoides Desf.

Sonchus hierrensis (Pit.) Boulos

Stachys arvensis (L.) L.

Stipa capensis Thunb.

\begin{tabular}{l} 
Trifolium angustifolium L. \\
\hline
\end{tabular}

Trifolium arvense L.

Trifolium campestre Schreber

Trifolium scabrum L. subsp. scabrum

Tuberaria guttata (L.) Fourr.

Urtica urens L.

Vicia angustifolia $\mathrm{L}$.

Vicia cirrhosa C. Sm. ex Webb \& Berthel

Vicia lutea L. subsp. Iutea

Vulpia myuros (L.) C.C. Gmelin

Wahlenbergia lobelioides (L. f.) Link subsp. lobelioides

\begin{tabular}{|c|c|c|c|c|c|c|c|c|c|c|c|c|}
\hline 11 & & & & 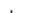 & 27 & 17 & & & & & & \\
\hline & & & & & & & 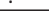 & & & & $\cdot$ & . \\
\hline & . & . & & & & + & 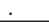 & . & & . &. & . \\
\hline+ & + & . & 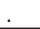 & + & . & . & + & + & + & . & . & + \\
\hline . & . & + & + & $\dot{0}$ & . & . & . & . & . & . & . & . \\
\hline . & . & . & & . & . & . & . & . & & . & . & . \\
\hline+ & + & . & 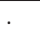 & . & + & . & 1.2 & 1.1 & 1.1 & . & . & . \\
\hline+ & . &. & . & 2.3 & 1.2 & 1.2 & . & . & . & . & . &. \\
\hline . & . & 1.1 & . & + & + & . & . & . & . & . & . & + \\
\hline . & . & . & + & . & . & . & . & . & . & 1.1 &. & . \\
\hline . & . & . & . & . & + & + & + & + & 1.1 & + & 1.1 & . \\
\hline 1.1 & 1.1 & . & + & . & . & . & 3.3 & 1.1 & 2.2 & . & + & . \\
\hline. &. & . & . & . & 1.1 & . & . & + & . & . &. & . \\
\hline . & . & + & + & + & . & . & . & . & . & . & . & . \\
\hline . & . & . & . & . & . & . & + & . & . & . & . & . \\
\hline . & . & 2.2 & . & . & . & . & . & 2.2 & 2.2 & . & . & . \\
\hline . & . & . & . & . & + & . & . & . & . & . & . & + \\
\hline . & . & . & + & . & . & . & . & . & . & . & . & . \\
\hline. &. &. & & + &. &. &. &. & - &. &. & + \\
\hline . & . & . & + &. & . & . &. & . & & . &. &. \\
\hline . & . & . & + & . & . & . & + & . & 1.1 & 1.1 & 1.1 & . \\
\hline+ & + &. & $\dot{.}$ & . &. &. & 1.1 & . &. & . &. &. \\
\hline+ & . & . & . & . & . & . & + & . & . & . & . & . \\
\hline . & . & 2.3 &. & . & 1.1 & 1.1 & 1.2 & 1.2 & . & . & . & . \\
\hline . & . & . & . & . & . & . & . & . & . & . & 2.2 & 2.2 \\
\hline. & + &. &. & . & . & . &. & . & . & . & + &. \\
\hline . & . & . & . & . & . & . & + & . & . & . & . & + \\
\hline . & . & . & 1.1 & . & . & . & . & . & . & 2.2 & 3.3 & . \\
\hline . & . & . & . & . & + & . & . & + & . & + & . & . \\
\hline 2.3 & 2.3 & 2.2 & 3.4 & 2.2 & 2.2 & 2.3 & 3.3 & 3.3 & 1.2 & 1.2 & 2.2 & 2.3 \\
\hline+ & . & 1.1 & . & 2.3 & 1.2 & . & . & . & . & . & . & 1.1 \\
\hline+ & + & . & . & . & . & . & + & 1.1 & 1.1 & . & . & . \\
\hline . & + & . & . & + & . & . & . & . & . & . & . & . \\
\hline+ & . & . & . & + & . & . & + & + & . & . & + & . \\
\hline . & . & . & . & . & + & + & . & + & . & . & . & . \\
\hline+ & 1.1 & . & . & . & + & + & . & . & 1.1 & . &. & . \\
\hline 2.3 & 1.2 & 1.1 & 2.3 & + & 1.1 & . & + & + & 3.3 & 2.2 & 3.3 & + \\
\hline . & . &. & . & . & + &. & . & . &. & . & . & . \\
\hline . & + & . &. & . & + & . & . &. & . & . &. &. \\
\hline 1.1 & . & . & . & . & . & . & . & 2.2 & . & . & . & . \\
\hline . & . & . & . & . & + & . & . & . & . & . & . & . \\
\hline . & . & . & . & + & 1.2 & 2.3 & . & . & . & . & . &. \\
\hline . & . & . & + & . & . & . & . & . & . & 1.1 & + & . \\
\hline . & . & . & . & . & + & . & . & . & . & . & . & + \\
\hline+ & 1.1 & + & . & . & + & . & 1.2 & . & + & . & . & . \\
\hline 1.1 & . & + & . &. & . & . & . & + & + & + & . & + \\
\hline . & . & . & . & + & + & . & . & . & . & . & . & . \\
\hline 1.1 & 1.1 &. & . & 2.2 & 2.2 & 2.2 & 2.3 & 1.1 & 1.2 & . & . & . \\
\hline 1.1 & . & 1.2 & . & 2.2 & 1.2 & . & 1.2 & 1.2 & 1.1 & . & . & 1.1 \\
\hline . & . & . & . & + & + & . & . & + & . & . & . & . \\
\hline . & . & . & . & 1.1 & 1.1 & 1.1 & . & . & . & . & . & . \\
\hline . & . & . & . & + & + & . & . & . & . & . & . & . \\
\hline . & . & 3.3 & + & 1.2 & 1.2 & . & . & . & 1.2 & . & 1.1 & 4.4 \\
\hline . & . & . & . & . & . & 2.2 & . & + & 1.2 & . & . & . \\
\hline 1.1 & 1.1 & . & . & . & + & . & 1.1 & . & . & . & + & . \\
\hline. & . & . & . & . & . & . & . & . & . & . & . & + \\
\hline . & 2.2 & 1.1 &. & + & 1.1 & . & . & + & . & . & . &. \\
\hline . & . & . & . & + & . & . & . & . & . & . & . & . \\
\hline . & . & + & + & . & . & . & . & . & . & 1.2 & 1.2 & . \\
\hline 1.1 & 1.1 & . & . & . & . & . & . & 1.1 & . & . & . & . \\
\hline 1.1 & 1.1 & . & 1.1 & + & . & . & . & + & 2.2 & 1.1 & 1.1 &. \\
\hline 1.1 & 1.1 & . & . & + & . & . & + & 1.1 & 1.2 & . & 1.1 & . \\
\hline 1.1 & . & . & + & . & + & . & + & + & 1.1 & 1.1 & 1.1 & . \\
\hline 1.1 & 1.1 & . &. & . & . & . & . & 1.1 & 1.1 & . & 1.1 &. \\
\hline . & $\dot{.}$ & . & & . & . & . & . &. & & . & . & + \\
\hline . & . &. & & . & & . & . & $\dot{\square}$ & & + & + & . \\
\hline . & $\dot{.}$ & . & & & & . & . & 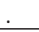 & & $\dot{.}$ & . &. \\
\hline+ & . & . & & . & . & . & . & . & . & . & . & . \\
\hline. & . & . & & & & + & . & 1.1 & & . & 1.1 & . \\
\hline . & . & . & 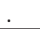 & . & . & . & . & . & . & . & . & + \\
\hline
\end{tabular}




\begin{tabular}{|c|c|c|c|c|c|c|c|c|c|c|c|}
\hline $\begin{array}{l}\text { P_18 } \\
{[14]}\end{array}$ & \begin{tabular}{|l} 
P_19 \\
{$[15]$}
\end{tabular} & $\begin{array}{l}\text { P_20 } \\
{[16]}\end{array}$ & $\begin{array}{l}\text { P_21 } \\
\text { [17] }\end{array}$ & $\begin{array}{l}\text { P_22 } \\
{[18]}\end{array}$ & $\begin{array}{l}\text { P_23 } \\
{[19]}\end{array}$ & $\begin{array}{l}\text { P_24 } \\
{[20]}\end{array}$ & $\begin{array}{l}\text { P_25 } \\
\text { [21] }\end{array}$ & $\begin{array}{l}\text { P_26 } \\
{[22]}\end{array}$ & $\begin{array}{l}\text { P_27 } \\
\text { [23] }\end{array}$ & $\begin{array}{l}\text { P_28 } \\
{[24]}\end{array}$ & {$[$ Syn] } \\
\hline . & . & . & . & . & . & . & . & . & . & . & 1 \\
\hline . & . & . & . & . & . & . & . & . & 1.1 & + & 1 \\
\hline+ & . & . & . & . & + & + & + & + & . & . & III \\
\hline . & . & . & . & . & . & . & . & + & . & . & 1 \\
\hline . & . & . & . & . & . & . & + & + & + & . & 1 \\
\hline . & 1.1 & . & . & + & . & . & . & . & . & . & II \\
\hline+ & . & . & . & + & . & . & . & . & . & . & II \\
\hline+ & . & . & + & . & . & . & . & . & . & 1.1 & II \\
\hline . & . & . & . & . & 1.2 & 1.1 & . & . & . & . & 1 \\
\hline . & . & + & . & . & + & . & + & + & . & . & IIII \\
\hline . & . & + & . & . & . & . & 1.1 & + & . & . & IIII \\
\hline . & . & . & . & . & . & . & . & . & . & 1.2 & 1 \\
\hline . &. & . & . & . & . & . & . & . & . & . & 1 \\
\hline+ & + & . & . & . & . & . & + & . & . & . & I \\
\hline 1.1 & 1.1 & 3.3 & 3.3 & 2.3 & . & . & . & . & . & 1.2 & II \\
\hline+ & . & . & . & . & . & . & . & . & . & + & I \\
\hline . & . & . & . & . & . & . & . & . & + & 1.2 & I \\
\hline+ & . & . & + & \begin{tabular}{|l|}
1.1 \\
\end{tabular} &. & . & . & . & . & . & II \\
\hline . & . & + & . & + & 1.2 & . & . & . & . & . & 1 \\
\hline . & . & . & . & . & . & . & . & . & . & . & II \\
\hline . & . & . & . & . & . & . & . & . & . & . & 1 \\
\hline . & . & . & . & . & . & . & + & . & . & . & I \\
\hline . & . & . & . & . & . & . & . & . & . & . & II \\
\hline 2.2 & \begin{tabular}{|l}
2.2 \\
\end{tabular} & 1.1 & 1.1 & 1.1 & . & . & + & . & II & 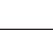 & \\
\hline . & . & . & . & . & . & + & . & + & . & . & 1 \\
\hline+ & . & . & . & . & . & . & . & . & . & . & I \\
\hline . & . & . & . & . & 1.1 & 3.4 & . & . & . & . & 11 \\
\hline . & . & . & . & . & + & . & . & . & . & . & 1 \\
\hline 4.4 & \begin{tabular}{|l|l|}
4.4 \\
\end{tabular} & 4.4 & 4.4 & 3.4 & 2.3 & 2.3 & 2.2 & 2.3 & 1.2 & 2.3 & $\mathrm{~V}$ \\
\hline 1.1 & 2.2 & + & 2.2 & 2.2 & . & . & . & . & 1.1 & + & III \\
\hline . & . & . & . & . & . & . & 1.1 & 1.1 & . & . & II \\
\hline . & & . & . & . & . & . & . & . & + & . & I \\
\hline . & 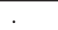 & . & . & . & . & . & . & . & . & . & II \\
\hline+ & 1.1 & + & . & + & . & + & . & . & 1.1 & . & II \\
\hline . & . & . &. & . & . & . & . & + & . & . & 11 \\
\hline . & + & . & . & + & 3.4 & . & 3.3 & 2.2 & 1.2 & + & IV \\
\hline+ & . & . & . & . & . & + & + & . & + & . & II \\
\hline . & . & . & . & . & . & . & . & . & . & + & 1 \\
\hline . & . & . & + & . & + & . & . & + & . & . & II \\
\hline . & + & + & . & 1.2 & . & . & + & + & 1.1 & . & II \\
\hline . & . & . & . & . & . & . & . & . & 1.1 & + & II \\
\hline . & 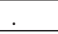 & . & . & . & + & + & . & . & . & . & II \\
\hline+ & . & . & . & . & + & + & . & . & . & + & II \\
\hline+ & 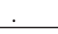 & + & . & . & . & . & + & + & . & . & III \\
\hline . & . & . & . & . & . & . & . & . & 1.2 & . & II \\
\hline+ & . & . & . & . & . & . & . & . & + & . & I \\
\hline . & . & . & . & . & . & . & 1.1 & 1.1 & 1.1 & 1.1 & IIII \\
\hline 1.1 & 3.3 & 2.2 & 1.2 & 1.1 & . & . & 1.1 & . & 1.1 & 2.2 & IV \\
\hline . & . & . & . & . & . & . & . & . & 1.1 & . & I \\
\hline . & + & 1.1 & . & . & . & . & . & . & 1.1 & 1.1 & II \\
\hline . & & + & . & . & . & . & . & . & . & . & 1 \\
\hline 2.2 & & . & + & . & . & . & . & . & + & . & III \\
\hline . & & . & + & + & . & . & . & . & . & . & 11 \\
\hline+ & . & . & . & . & + & + & + & 1.1 & 1.1 & . & III \\
\hline+ & . & . & + & . & . & . & . & . & . & . & I \\
\hline . & + & + & . &. & . & . & . & + & 1.1 & 1.1 & III \\
\hline . & . & . & . & . & + & + & + & + & + & . & II \\
\hline . & . & . & . & . & . & . & . & . & . & . & I \\
\hline . & . & . & . & . & . & + & . & . & . & . & 1 \\
\hline . & . & . & . & + & + & 1.1 & . & . & 1.1 & . & III \\
\hline . & . & . & . & . & . & 1.1 & + & . & + & + & IIII \\
\hline & & & & & & + & . & . &. & . & II \\
\hline & & & & & + & 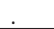 & . & + & . & . & II \\
\hline+ & + & . & + & 1.1 & . & . & . & . & . & . & II \\
\hline$\dot{.}$ & . & . & . & 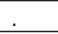 & 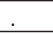 & 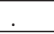 & . &. & . & + & 1 \\
\hline$\dot{.}$ & + & . & + & + & . & + & + & . & . & . & II \\
\hline . & . & . & . & . & . & . & + & + & . & + & 1 \\
\hline+ & . & . & . & . & + & . & . & + & . & . & II \\
\hline+ & + & . & + & + & . & + & + & . & 2.2 & . & II \\
\hline
\end{tabular}

Taxons with constancy lower than 3:

Aeonium hierrense (RP Murray) Pit \& Proust in 23(+) 24(+) Allium subvillosum Salzm. ex Schultes \& Schultes fil. in 24(+) Androcymbium hierrense A. Santos in $9(+)$

Andryala pinnatifida Ait. in 1(+) $9(+)$

Anthoxanthum aristatum Boiss. subsp. aristatum in 2(1.1) 24(+)

Asparagus umbellatus Link in 24(+)

Asterolinon linum-stellatum (L.) Duby in 2(+)

Bidens pilosa L. in 22(+)

Briza maxima $\mathrm{L}$. in $1(+) 2(1.1)$

Calendula arvensis L. in 21(+)

Carduus baeocephalus Webb subsp. microstigma Gaisberg \&

Wagenitz in $17(+) 18(+)$

Ceballosia fruticosa (L. f.) G. Kunkel in 13(1.1)

Centaurium tenuiflorum (Hoffmanns. \& Link) Fritsch subsp. tenuiflorum in $10(+)$ 20(+)

Cerastium glomeratum Thuill. in 24(+)

Convolvulus althaeoides L. subsp. althaeoides in 21(+)

Cosentinia vellea (Aiton) Tod. in 11(+) 12(+)

Crassula tillaea Lester-Garland in 17(+) 22(+)

Crepis foetida L. subsp. foetida in 2(+) 7(+)

Ebingeria elegans (Lowe) Chrtek. \& Krisa in 2(1.1) 24(1.1)

Echium aculeatum Poir. in 21(+) 22(+)

Erica arborea L. in 24(+)

Erodium botrys (Cav.) Bertol. in 14(+) 21(+)

Erodium chium (L.) Willd. subsp. chium in $22(+)$

Erysimum bicolor (Hornem.) DC. in 23(+)

Foeniculum vulgare Mill. subsp. vulgare in 24(+)

Galium aparine L. in 24(+)

Geranium purpureum Vill. in 24(+)

Gnaphalium luteo-album L. in 4(+) 5(+)

Habenaria tridactylites Lindl. in 24(+)

Hippocrepis multisiliquosa L. in 11(+) 21(+)

Hirschfeldia incana (L.) Lagreze-Fossat in 1(+) 21(+)

Hypericum canariense L. in 23(2.2) 24(2.3)

Hypochoeris glabra L. in $20(+) 22(+)$

Lamarckia aurea (L.) Moench in 23(+)

Lathyrus tingitanus $\mathrm{L}$. in 7(+)

Limonium pectinatum var. solandri (Webb \& Bertel.) Kuntze in $13(+)$

Lolium canariense Steud. in 8(1.1) 22(+)

Lotus sessilifolius DC. in 7(1.1)

Marrubium vulgare L. in 22(+)

Mercurialis canariensis Obbard \& S.A. Harris in 21(+)

Nicotiana glauca R.C. Graham in 18(+)

Orobanche minor $\mathrm{Sm}$. in $2(+)$

Parietaria debilis Forster fil. in 17(+) 18(+)

Pinus canariensis Sweet ex Sprengel in 12(1.2) 20(+)

Plantago afra L. in 3(+) 14(+)

Pteridium aquilinum (L.) Kuhn in 23(1.2)

Reichardia ligulata (Vent.) G.Kunkel \& Sunding in 6(+) 23(+) Rumex angiocarpus Murb. in 23(+)

Scandix pecten-veneris L. subsp. pecten-veneris in $22(+)$

Selaginella denticulata (L.) Link in 24(2.3)

Senecio teneriffae Sch. Bip. ex Bolle in 21(+)

Sherardia arvensis $\mathrm{L}$. in 2(+)

Silene vulgaris (Moench) Garcke in 22(+) 24(+)

Solanum nigrum L. subsp. nigrum in $8(+)$

Sonchus oleraceus L. in 15(1.1)

Sonchus tenerrimus L. in 8(+) 21(+)

Stellaria media (L.) Vill. subsp. media in 22(+)

Tolpis umbellata Bertol. in 12(+)

Trifolium glomeratum L. in 21(+) 22(+)

Trifolium pratense $\mathrm{L}$. in 1(1.1)

Trifolium subterraneum L. in 8(+)

Umbilicus horizontalis (Guss.) DC. in 14(+) 18(+) 


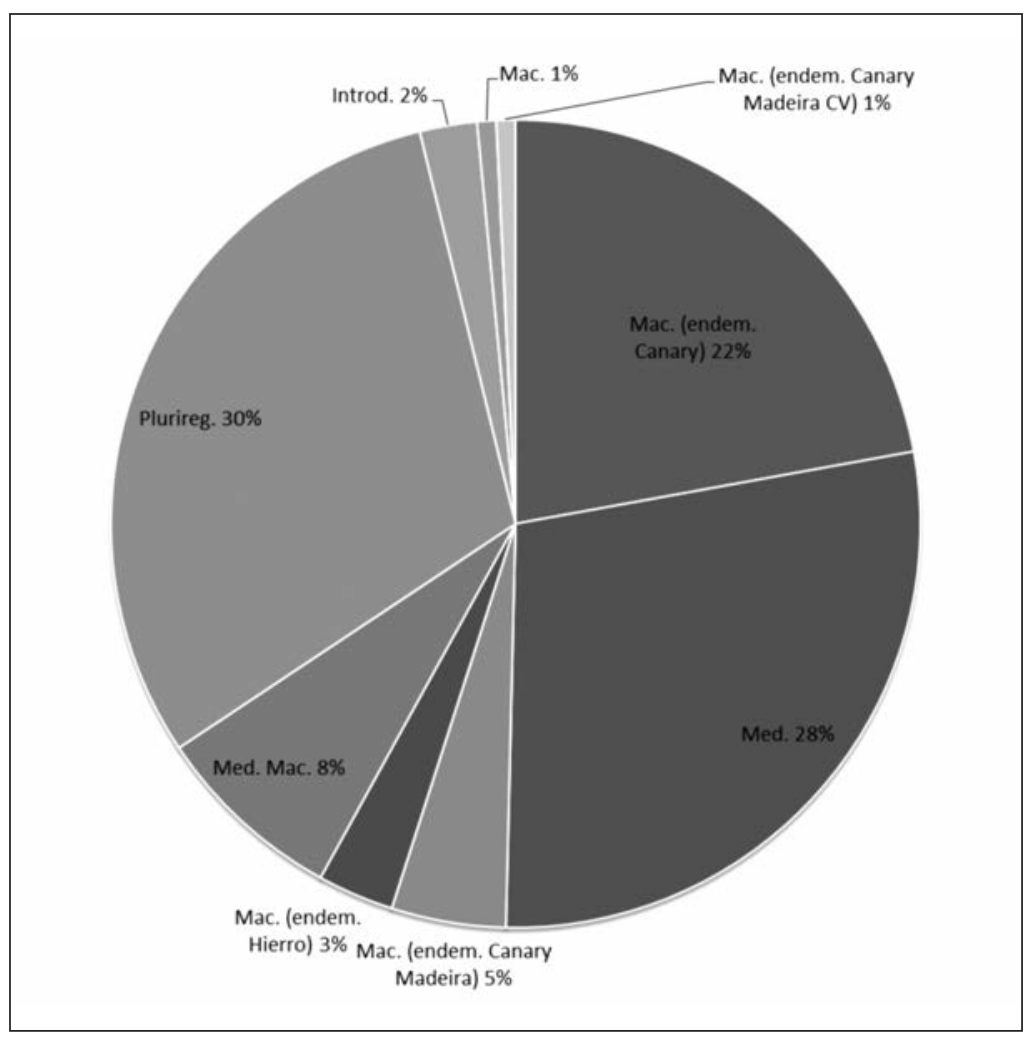

Figure 2 - Chorology of the taxa living in the juniper woodlands. Mac.: endemic from Macaronesia floristic biogeographic region as defined by Tahtajan (1986); endem.: endemic from Canary Isles, Madeira Islands; CV: Cape Verd Isles; Med.: Mediterranean floristic biogeographic region as defined by Tahtajan (1986); Introd.: introduced; Plurireg.: pluriregional.

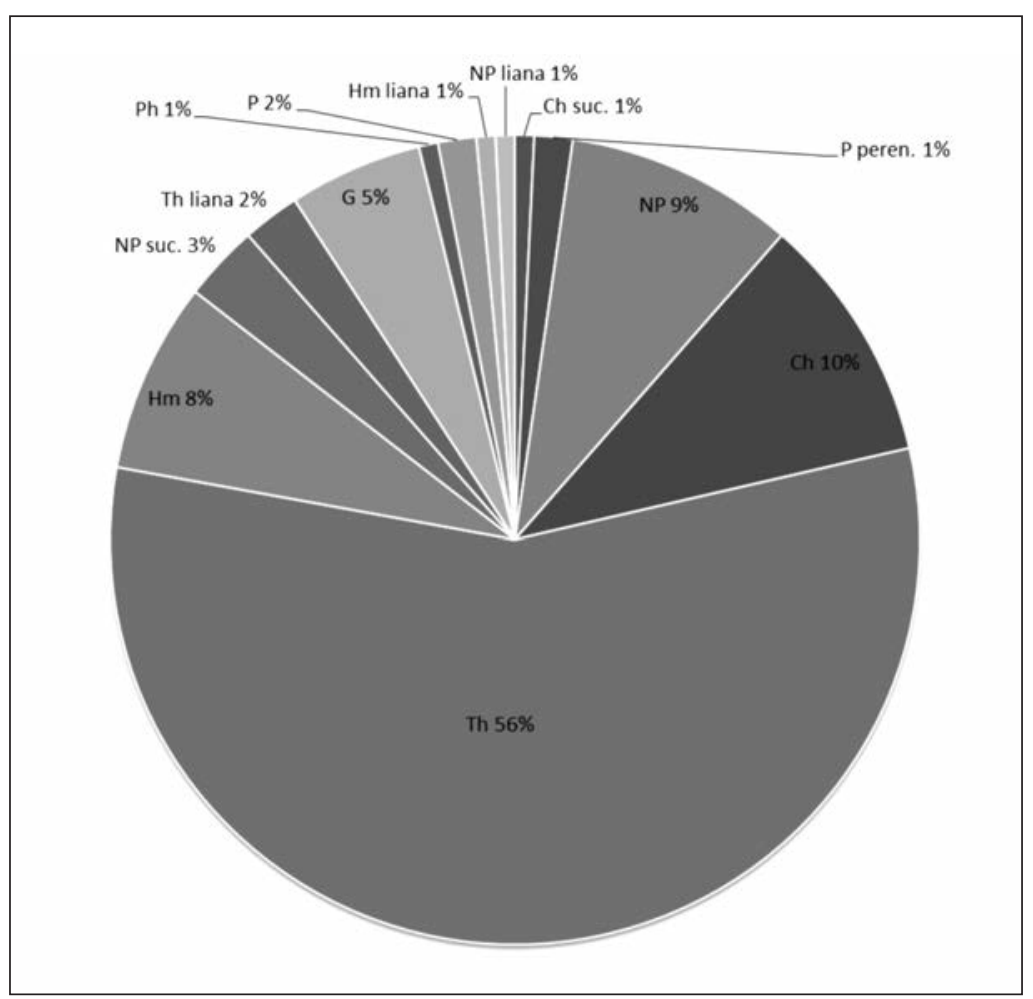

Figure 3 - Life forms of the taxa living in the juniper woodlands. (Ch: chamaephytes; G: geophytes; Hm: Hemicryptophytes; liana: lianas; NP: nanophanerophytes; peren.: perennifolious; Ph and P: Phanerophytes; suc.: succulent; Th: Therophytes). total 550 taxa found on the island (Stierstorfer \& von Gaisberg 2006) have been detected in these juniper woodlands.

The associated flora of these juniper woodlands makes up $30.6 \%$ of the endemic taxa from the Macaronesian floristic region, such as defined by Tahktajan (1986), of which $22.1 \%$ are Canary island endemics, $4.6 \%$ are endemics from the Canary and Madeira archipelagos, $3.1 \%$ are endemics from El Hierro, and $0.8 \%$ endemics from the Canary Isles, Madeira and Cape Verde (Figure 2). A second group of taxa is formed by species of a pluriregional distribution, with a $30.5 \%$ representation. The Mediterranean taxa are represented by a further $28.2 \%$. Another portion, $7.6 \%$, is made up of plants living in the Mediterranean and Macaronesia. In final place, the allochtonous taxa growing in the juniper woodlands are reduced to a mere $2.3 \%$.

The biodiversity analysis, using cluster analyses UPGMA method and Chord coefficient for the different studied plots, reflects a great affinity between the plots located in the low areas, on the one hand, and those located in the more elevated areas on the other. From these latter ones we can distinguish the plots located in wetter environmental conditions, near the laurel forest (plots $P_{-} 05, P_{-} 13$; $P_{-}$14), from those located near the Canary pine Pinus canariensis (plots $\mathrm{P}_{-}$08, $\mathrm{P}_{-} 15$; $P_{\text {_}}$ 16), zone, in drier environmental conditions. There also exist differences between the plots located on northern exposed slopes ("barlovento" in Spanish), which receive the influence of the wet winds (air masses a of a very humid nature) and the plots located on southern sheltered slopes "sotavento", where the descending air is drier.

The number of species on the studied plots oscillates between 14 and 33 taxa. We have observed two maximums in the decreasing number as the altitude descends; one between 200 and $400 \mathrm{~m}$ above sea level, and another between 500 and $800 \mathrm{~m}$. The slope does not seem to greatly affect the number of species, but orientation, however, has a great influence. The highest biodiversity is observed on the plots with a $\mathrm{N}$ and NNE aspect, which have a correlation with the northern winds "alisos" ("alisios" in Spanish). These winds model the aerodynamic forms of the adult individuals sampled (Salvà-Catarineu \& Romo 2008). A second block, with remarkable but slightly less biodiversity, is observed 
in the plots with a south-facing aspect. No stands of juniper woodland have been observed on the E or SW aspects (Figure 4).

The dendrogram reflects the affinity between the dry juniper woodlands from the lowlands and the others situated in more elevated stations (Figure 5). From these we can differentiate those situated in moister conditions, which enter in contact with laurel forests on one hand, and those in close proximity with the Pinus canariensis forest, on the other.

\section{Discussion}

The obtained results are comparable to the results provided by Fernández Palacios et al. (2008) for Tenerife and La Gomera islands. But their approach was somewhat different, and therefore their results, in particular for the island of Tenerife, because the therophytes were not taken into account.

Bearing this in mind, when it comes to introduced plants, Fernández Palacios et al. (loc. cit.) give an amount of $14 \%$, as opposed to our $2.3 \%$. This lower value can be explained by the much reduced anthropic impact on the natural vegetation of El Hierro and by the presence of a lower number of habitats opened to invasive plants on this island.

The globally widespread species, that is, the pluriregional ones, make up 29\% in Fernández Palacios et al.'s survey, and we have found a similar value $30.5 \%$, for El Hierro.

These authors detected $48 \%$ of macaronesian endemics, whereas we found only $31 \%$. Finally Fernández Palacios et al. gave an amount superior to $20 \%$ for the Canary endemics, as opposed to the $25.2 \%$ detected by us. All these values could be explained by the reduced surface of El Hierro, as compared with Tenerife $\left(2.034 \mathrm{~km}^{2}\right)$.

In relation to the life forms, the elevated number of therophytes here is remarkable (Figure 3). These make up more than a half of the species $(56 \%)$. There is a lack of comparable data from other Canary islands, since the annuals were not studied by these authors. Nevertheless, this elevated number of annuals can be explained by the structure of this plant community, which is formed by woodland patches that contain gaps, with a very shallow soil, in which only therophytes can grow.

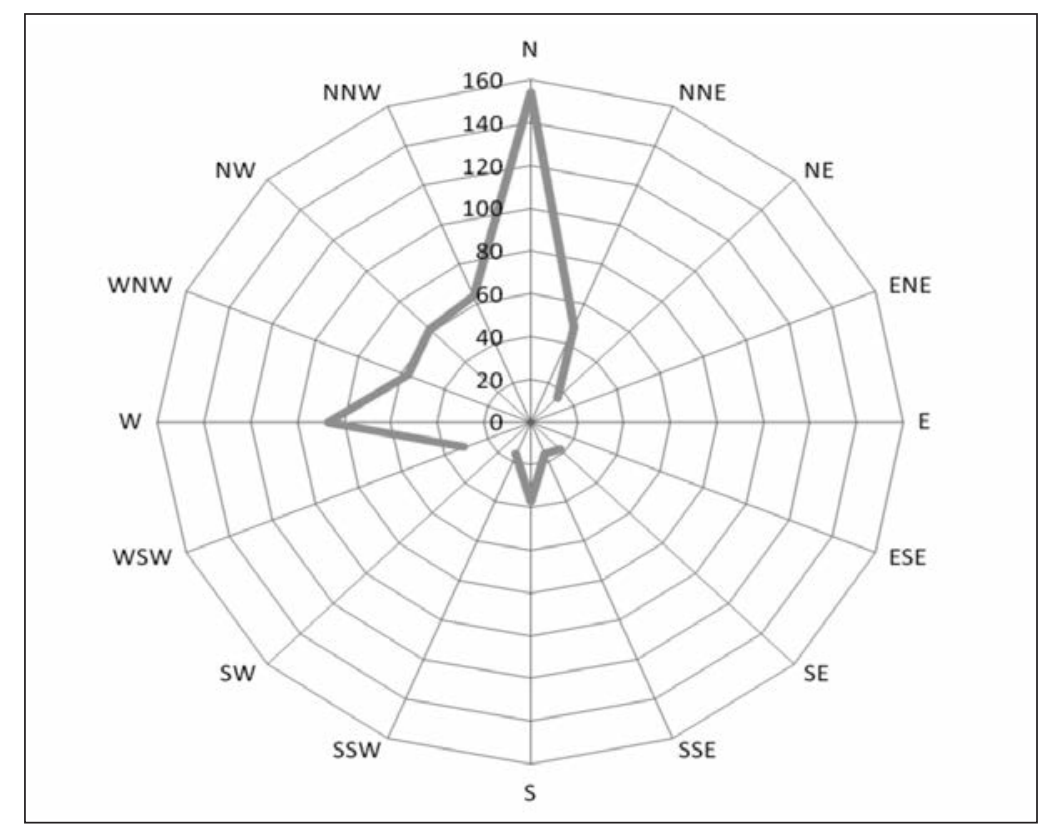

Figure 4 - Relation between number of taxa and aspect.

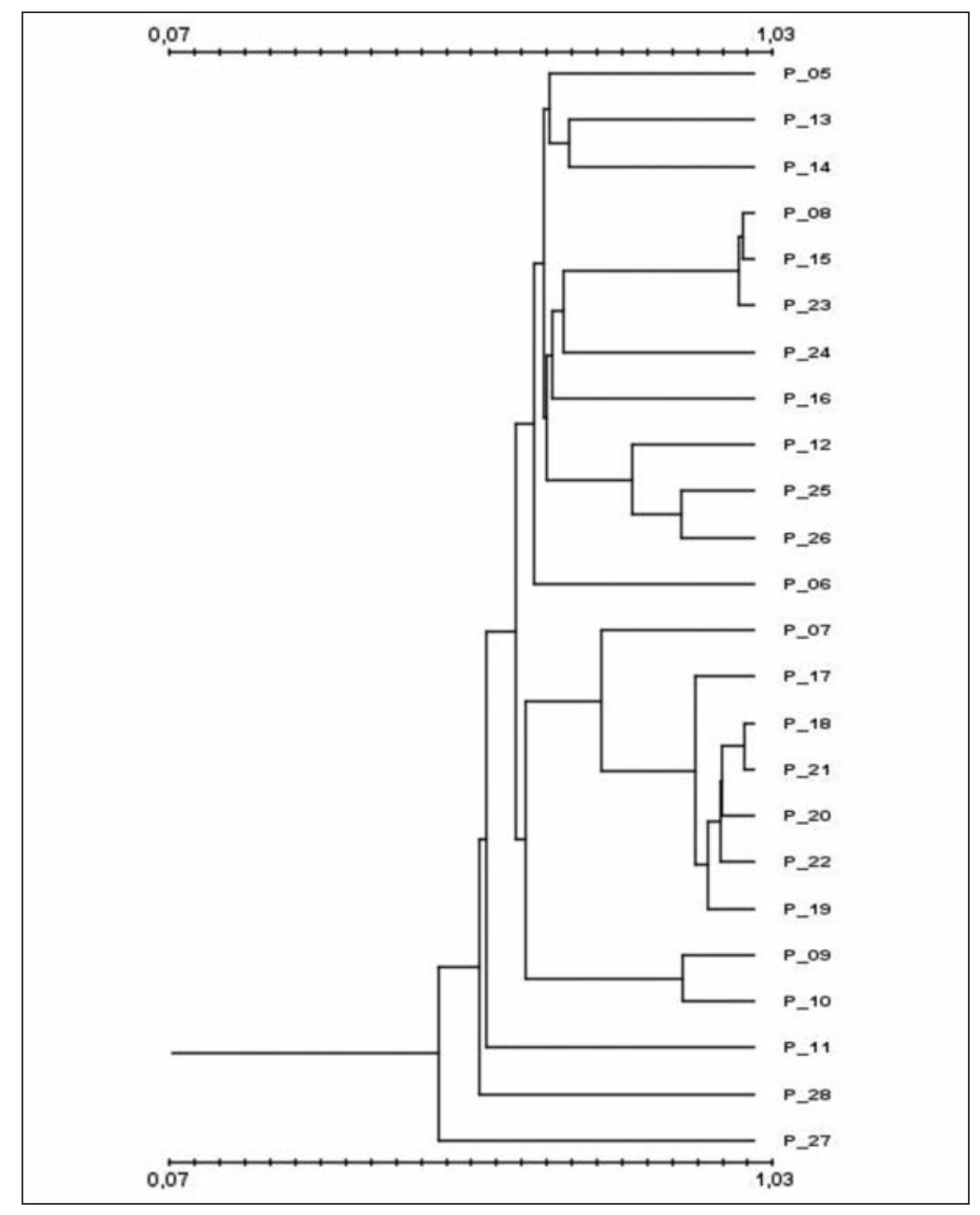

Figure 5 - Dendrogram that reflects the floristic affinities between the plots studied. Cluster analyses using UPGMA method and Chord coefficient. $P \_05$ to $P \_14$ are wet Canarian juniper woodlands from el Golfo area; $P \_08$ to $P \_06$ are dry Canarian juniper woodlands from el Julán area; $P \_07$ to $P \_10$ are dry Canarian juniper woodlands from the Sabinosa area; $P_{-} 11$ to $P \_27$ are dry Canarian juniper woodlands from the El Sabinar area. To see the correspondence between code plots and relevées see Table 1. 
The phanerophytes, including the different nanophanerophytes, constitute a $52.34 \%$, and the chamaephytes a $20.9 \%$ (the therophytes have been eliminated in order to compare our data with that of Fernández Palacios et al. [loc. cit.]). In other words, woody plants represent $73.24 \%$ of the total and perennial non-woody species make up $26.76 \%$ of the total. Together, greater values are obtained for the studied plots on El Hierro. This can be explained by the better state of conservation of the natural vegetation on this small island.

Concerning the number of species (Table 1), and taking into account the fact that in Fernández Palacios et al. the plots were of $314 \mathrm{~m}^{2}$ and our plots are of $200 \mathrm{~m}^{2}$, the number of taxa oscillates between 14 and 33 obtained by us and $9-41$ by Fernández Palacios et al. This difference is considered to be of little significance. Prior to this, Gaisberg (2005) obtained between 9 and 49 taxa for plots whose surface oscillated between 10-20 m², del Arco et al. (1996) recorded a range of 4-21 taxa for plots of $100 \mathrm{~m}^{2}$, and Hernández (1987) obtained between 4 and 8 species for plots that had between 25 and $100 \mathrm{~m}^{2}$ of surface area.

\section{Conclusion}

The juniper woodlands from El Hierro island exhibit a high degree of biodiversity. This phytodiversity richness seems more related to the physical properties of the sampled localities than to general environmental conditions (Salvà-Catarineu et al. 2012). These results are congruent with the ones obtained by Box \& Fujiwara (2011) working on North American vegetation.

These open woodlands should be considered as hotspots within the greater hotspot of the Canary islands. Within them, Salvà-Catarineu \& Romo (2008) observed practically no regeneration, and therefore we conclude that measures to preserve and manage them should be urgently drawn up.

The protection of areas within El Hierro such as the "Parque Rural de Frontera and La Reserva Integral de Mencafate", that are SCI (Sites of Community Importance, as defined by the European Commission Habitat Directive, 92/43/EEC) which contain Canarian juniper formations, is a welcome measure that reinforces the need for new studies on this type of open woodland dynamics.
Moreover, in our opinion, these dry woodlands - located in an Oceanic archipelago are especially sensitive to climatic change, which in itself justifies the establishment of study programmes based on changes in their biodiversity over an extended period of time.

\section{Acknowledgements}

To the Island authorities "Cabildo Insular del Hierro", for facilitating access to protected areas such as the Reserva de Mencáfate and the Julán. Especially to Claribel González and Miguel Angel Rodríguez. who helped improve information and documentation for these areas. Also to Montse Granados and Quirina Linares for their help during the fieldwork. This project was funded by Ministerio de Ciencia e Innovación-Spain (CSO201124425) and from our own resources. Two anonymous contributers have improved this paper with their criticism and advice.

\section{References}

Blondel J., Aronson J., Bodiu J.Y. \& Boeuf G., 2010. The Mediterranean Region: biodiversity in space and time. Oxford, Oxford University Press, 376 p.

Box E.O. \& Fujiwara K., 2011. Sorting plots not taxa for studying vegetation structure and plant species richness. Plant Biosystems 145: 46-53.

Braun-Blanquet J., 1979. Fitosociología. Madrid, Ed. Blume, $820 \mathrm{p}$.

De Cáceres M, Font X., García R. \& Oliva F., 2003. B-VegAna, un paquete de programas para la gestión y análisis de datos ecológicos. VII Congreso Nacional de la Asociación Española de Ecología Terrestre. Barcelona, Julio, 2003 : 1484-1497.

Del Arco M., Acebes J.R. \& Pérez de Paz P.L., 1996. Bioclimatology and climatophilous vegetation of the island of Hierro (Canary Islands). Phytocoenologia 26: 445-479.

Fernández Palacios J.M., Otto R., Delgado J.D., Arévalo J.R., Naranjo A., González-Artiles F., Morici C., Barone R., 2008. Los bosques termófilos de Canarias. Excmo. Cabildo Insular de Tenerife, Santa Cruz de Tenerife, 192 p.

Fuster J.M., Herrán F., Cendrero A., Coello J., Cantagrel J.M., Ancochea E. \& Ibarrola E., 1993. Geocronología de la isla de el Hierro (Islas Canarias). Bol. R. Soc. Esp. Hist. Nat. Sec. Geol. 88 : 85-97.

Hernández C., 1987. Flora y vegetación liquénica epifítica de los sabinares Herreños. Bibliotheca Lichenologica, 27. Berlin-Stuttgart, J. Cramer, 260 p.

Médail F. \& Quézel P., 1999. Biodiversity hotspots in the Mediterranean Basin. Setting global Conservation Priorities. Conservation Biology 13: 15101513. 
Myers N., Mittermeier R.A., Fonsecay G.A.G. da \& Kent J., 2000. Biodiversity hotspots for conservation prioriries. Nature 403: 835-858.

Nogales N., Padrón B., Padilla D.P. \& Nieves C., 2009. Germination patterns throughout an insular altitudinal gradient: The case of the Macaronesian endemic plant Rubia fruticosa Ait. (Rubiaceae) in El Hierro (Canary Islands). Flora 204: 543-548.

Otto R., Krüsi B.O., Schaffner S., Meuwly P., Delgado J.D., Arévalo J.R. \& Fernández-Palacios J.M., 2006. Ecología, estructura y dinámica de las poblaciones de la sabina canaria (Juniperus turbinata ssp. canariensis) en Tenerife y la Gomera. In: Actas del III Colloquio Internacional Sobre los Sabinares y Enebrales (Género Juniperus): Ecología y Gestión Forestal Sostenible, Soria, 24 a 26 de Mayo 2006, Tomo $1:$ 151-159.

Salvà-Catarineu M. \& Romo A., 2008. Uso de TIG para la conservación del sabinar de Juniperus turbinata subsp. canariensis en la isla de el Hierro. In: Hernández L. \& Parreño J.M. (eds), Tecnologías de la información geográfica para el desarrollo territorial. Servicio de Publicaciones y Difusión Cientí- fica de la ULPGC, Las Palmas de Gran Canaria : 766-776.

Salvà-Catarineu M., Romo A. \& Salvador Franch F., 2012. «Estructura de edad y biodiversidad de los sabinares de Juniperus turbinata Guss. en El Hierro (Islas Canarias) ». In: Cunill R., Pèlacs A., PérezObiol R. \& Soriano J.M. (eds), Las zonas de montaña: gestión y biodiversidad. Sant Pere de Ribes, Ed. Falcó : 40-45.

Santos A., 1980. Contribución a la flora y a la vegetación de la isla de El Hierro (Islas Canarias). Fundación Juan March, Serie Universitaria 114, Madrid, $51 \mathrm{p}$.

Stierstorfer C. \& von Gaisberg M., 2006. Annotated check-list and distribution of the vascular plants of El Hierro, Canary Islands, Spain. Englera 27: 1-221.

Takhtajan A., 1986. Floristic regions of the world. University of California Press, Berkeley, California, $522 \mathrm{p}$.

Von Gaisberg M., 2005. Die vegetation der Fussstufe von El Hierro (Kanarischen Inseln). Dissertationes Botanicae 395: 1-364. 